\title{
The importance of following the evolution of the dust in galaxies on their SEDs
}

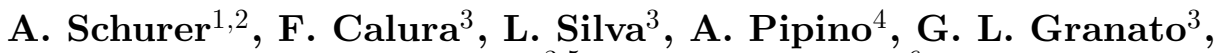 \\ F. Matteucci ${ }^{3,5}$ and R. Maiolino ${ }^{6}$ \\ ${ }^{1}$ Astrophysics Sector, SISSA/ISAS, Via Beirut 2-4, I-34014 Trieste, Italy \\ email: schurer@sissa.it \\ ${ }^{2}$ INAF, Osservatorio Astronomico di Padova, Vicolo dell'Osservatorio 5, I-35122 Padova, Italy \\ ${ }^{3}$ INAF, Osservatorio Astronomico di Trieste, Via G. B. Tiepolo 11 I-34131 Trieste, Italy \\ ${ }^{4}$ Astrophysics, University of Oxford, Denys Wilkinson Building, \\ Keble Road, Oxford OX1 3RH, UK \\ ${ }^{5}$ INAF, Dipartimento Astronomico-Universita di Trieste, \\ Via G. B. Tiepolo 11 I-34131 Trieste, Italy \\ ${ }^{6}$ INAF Osservatorio Astronomico di Roma, via di Frascati 33, Monte Porzio Catone, Italy
}

\begin{abstract}
We present photometric evolution of galaxies in which, in addition to the stellar component, the effects of an evolving dusty interstellar medium have been included with particular care. Starting from the work of Calura, Pipino \& Matteucci (2008), in which chemical evolution models have been used to study the evolution of both the gas and dust components of the interstellar medium in spiral, elliptical and irregular galaxies, it has been possible to combine these models with a spectrophotometric stellar synthesis code that includes dust reprocessing (GRASIL) (Silva 1998) to analyse the evolution of the Spectral Energy Distributions (SED) of these galaxies. We test our models against observed SEDs both in the local universe and at high redshift. The importance of following the dust evolution is investigated by comparing our results with those obtained by adopting simple assumptions to treat this component. Possible errors from assuming a Milky Way dust composition and a dust-to-gas ratio scaling with the metallicity particularly in young galaxies, ellipticals and low metallicity galaxies are highlighted.
\end{abstract}

Keywords. ISM: abundances, ISM: dust, extinction, galaxies: abundances, galaxies: evolution

\section{Overview}

The presence of dust particles in the interstellar medium (ISM) of galaxies has been shown to have a significant effect on their spectral energy distribution (SED). Indeed it has been inferred from measurements of the FIR/sub-mm background that approximately $\sim 50$ per cent of star light has been reprocessed by dust over the entire history of the universe (see e.g. Hauser \& Dwek 2001 for a review). Hence, a proper treatment of the dust reprocessing in galaxies is essential, both to derive physical quantities such as starformation rates and star-formation histories from observations and also when testing theoretical galaxy formation models against observations. Several different models have been proposed with varying degrees of sophistication to treat the effect of the dust on the SED (see e.g. Popescu \& Tuffs 2005 for a review).

A correct treatment of dust reprocessing is however complex and generally will contain several free parameters. Hence several processes, including the effect of the evolution of the dust component, are often overlooked for simplicity. As a result, it is common to assume that the chemical composition of the dust does not vary from that inferred from observations from within the solar neighbourhood and, where the total mass of dust grain 
is required, often it is related to the metallicity and mass of the gas component via a simple scaling relation (e.g. Granato et al. 2000).

We have tested the importance of following the evolution of the dust component on the SED of galaxies of three different morphologies. We have combined the chemical and dust evolution model of Calura, Pipino \& Matteucci (2008) (hereafter CPM08), which follows the chemical and dust grain evolution of galaxies of different morphology, with the GRASIL code (Silva et al. 1998, hereafter S98) which used the calculated stellar and dust evolution, in order to calculate the SED expected for the galaxy. The importance of following the dust evolution is tested by comparing these fiducial SEDs with SEDs generated for galaxies with the same chemical evolution, but instead of using the predictions from the dust evolution model as before, two common simplifications are adopted instead:

- that the ratio of the dust mass to gas mass is proportional to the metallicity through a constant normalised to the Milky Way. So dust mass $\propto$ metallicity $\times$ gas mass.

- that the chemical composition does not evolve. So that the fraction of carbon to silicate will be set to the value 0.19 for all times and morphologies (a theoretical value found by CPM08 for the ratio of C/Si at 13 Gyr in the solar neighborhood model).

Many examples of the use of the GRASIL code can be found within the literature (e.g. S98, Panuzzo et al. 2007, Iglesias-Pramo et al. 2007, Vega et al. 2008) and this means that, although there are several other 'free' parameters within the code, a suitable value for all these parameters can be taken from the previous works.

By conducting a quantitative comparison between the SEDs generated using the fiducial model and those generated adopting the two simplifications, it is possible to estimate what errors could theoretically be expected from adopting the above assumptions in each of the galaxies of different morphological type.

\section{Comparisons to Observations}

To test the validity of our fiducial models, they are compared, where possible, with observed SEDs.

\subsection{Spiral Galaxies}

Figure 1a shows the results for the fiducial theoretical SEDs generated at 12 Gyrs for the spiral model compared to the mean SED of spiral galaxies of types $\mathrm{SBb}$ to $\mathrm{SBc}$ and SAb to SAc from the SINGS sample (Dale et al. 2007). The theoretical model shows a good correlation with the observed spiral galaxies.

\subsection{Irregular Galaxies}

Figure 1b shows the results for the theoretical SEDs generated at 12 Gyrs compared to the SEDs for all the magellanic type irregular galaxies of the SINGS sample (Dale et al. 2007). Although the irregular galaxies show some scatter in their SEDs, the theoretical model fits the average SED shape remarkably well. Also shown in the plot is the SED generated if the two dust assumptions outlined in the overview are adopted to describe the evolution of the dust (instead of the full CPM08 treatment). The adoption of the two assumptions leads to a clear discrepancy between the two SEDs, particularly in the IR and UV. Although still largely consistent with the observed SEDs, it seems that the simplified dust treatment is leading to more dust reprocessing. A common measure of the quantity of dust reprocessing in a galaxy is the observed IR/UV ratio (e.g. Dale et al. 2007). Using the same formalism as Dale et al. (2007) and equation 4 from Dale \& Helou (2002) the median Total IR to UV ratio was calculated for the irregular galaxies 

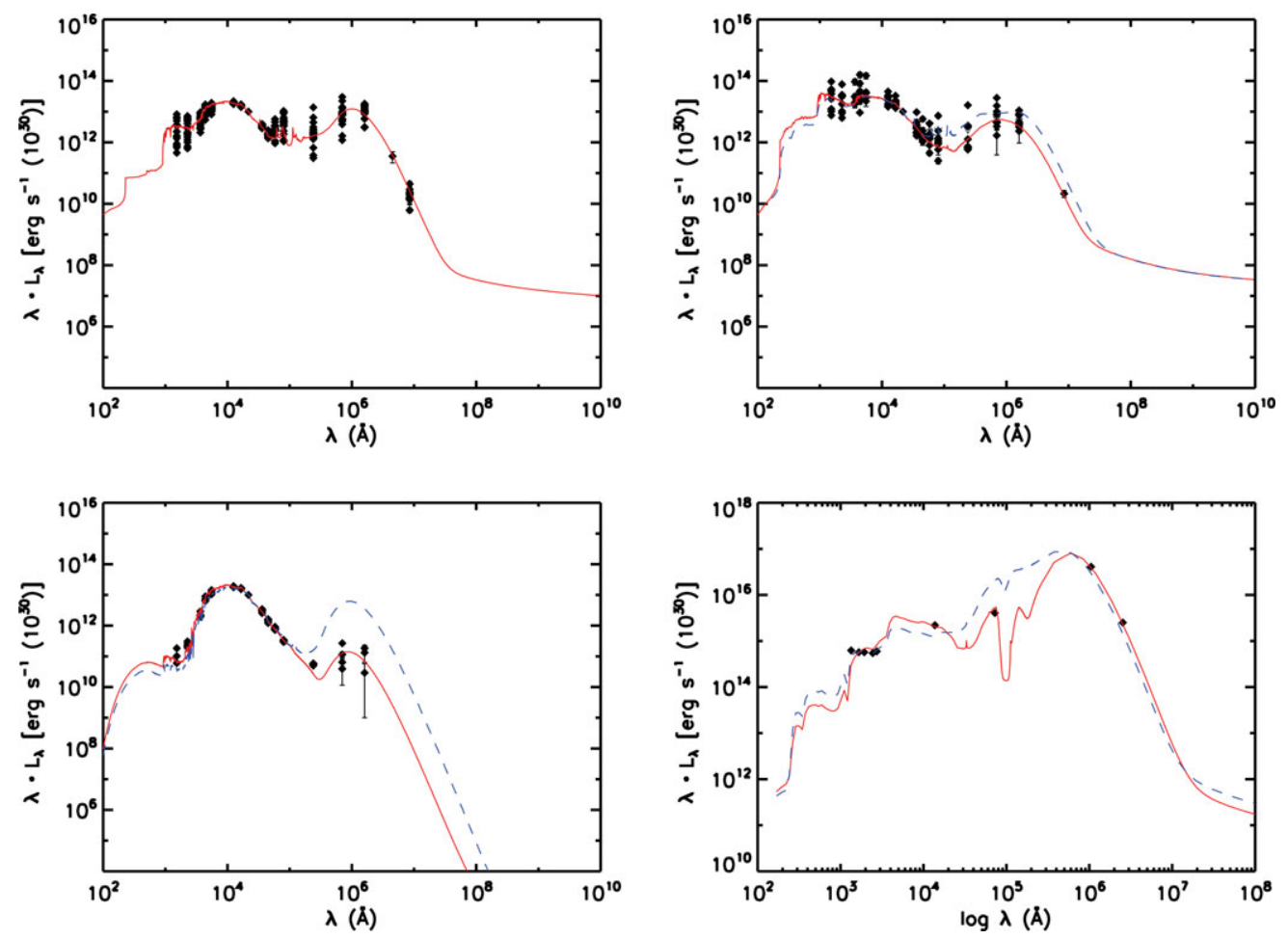

Figure 1. Comparison of the fiducial theoretical models at 12 Gyrs with the SEDs from all galaxies of the particular morphological type in the SINGS sample, normalised to the $K$-band, for (a) spiral galaxies, (b) irregular galaxies, (c) elliptical galaxies. (d) shows a comparison between the theoretical SED of the elliptical galaxy at 0.65 Gyrs with a SCUBA galaxy from the SHADES sample. Solid line (red) - Full CPM08 model. Dashed line (blue) - Same chemical evolution but adopting assumptions for the dust component.

in the sample to be 0.22 agreeing well with the value of 0.21 calculated for the fiducial CPM08 model. For the model with the two assumptions, however a value of 0.67 was calculated, implying that too much energy has been reprocessed from the UV to the IR.

\subsection{Elliptical Galaxies}

Figure 1c shows the result for the theoretical SED of the elliptical model (model La1 in CPM08) generated at 12 Gyrs compared to the SEDs of the four local elliptical galaxies from the SINGS sample (Dale et al. 2007). The SED generated by the fiducial model matches the observations well. However, if the dust evolution is not followed and the two dust assumptions are adopted instead, SEDs are generated which significantly overestimate the amount of reprocessing and are inconsistent with the observed galaxies.

Our results for the elliptical galaxy have also been compared to SCUBA galaxies at high redshift. These galaxies are believed to represent the birth of giant elliptical galaxies (Lilly et al. 1999, Eales et al. 2000). Figure 1d shows the SED of the elliptical model at an age of 0.65 Gyr compared to a SCUBA galaxy from the SHADES sample (Clements et al. 2008). In the plot the SCUBA galaxy is shown at a redshift of 2.30 , the same redshift as found in Clements et al. (2008). The fit is good for the fiducial model. However if instead of following the dust evolution the two assumptions are used the SEDs generated are inconsistent with the observed SEDs. 

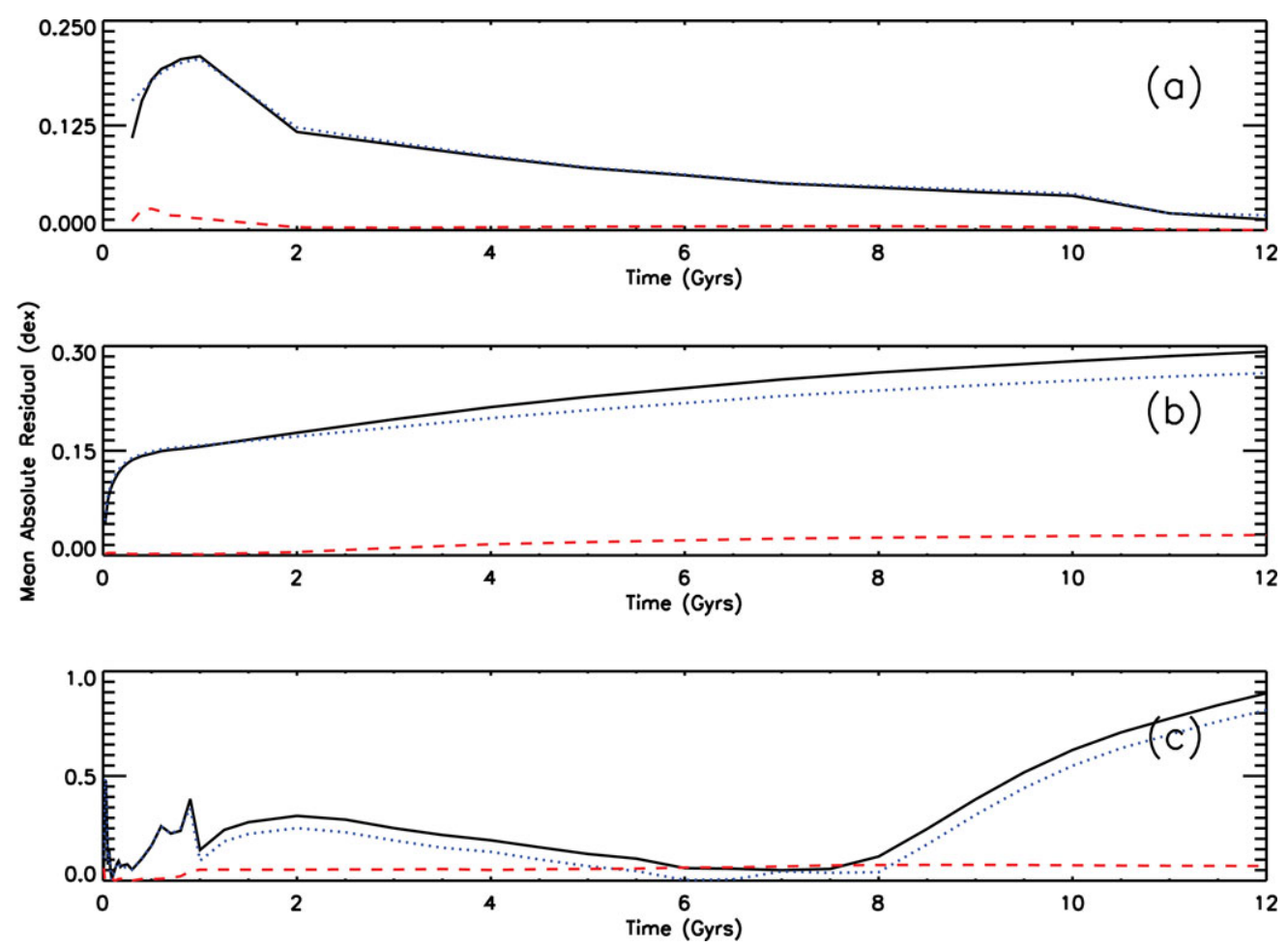

Figure 2. Absolute mean residual between the fiducial CPM08 models and models with the chemical evolution but adopting assumptions for the dust component. Thick Line (black) Adopting both assumptions. Dotted line (blue) - Assumed dust-to-gas ratio, chemical composition calculated by CPM08. Dashed line (red) - Assumed chemical composition, dust-to-gas ratio calculated by CPM08. For (a) spiral galaxies, (b) elliptical galaxies, (c) irregular galaxies.

\section{Implications}

In this work only one likely chemical and dust evolution model, with one star-formation history, has been followed for each morphological type of galaxy based on average properties of the galaxy type. It is therefore not possible to prove conclusively the accuracy of the models, since it is unlikely that they will match exactly the SED of any one specific galaxy. However, since the chemical-evolution models are designed to match average properties, the generated SEDs would be expected to match the general appearance of many galaxies of the specific type modeled and this is indeed what has been found. Plots have been presented which show acceptable fits to local spirals, irregulars and ellipticals simultaneously in their starbursting and passive phases. It should also be noted that although both models contain many parameters, very little fine tuning has been performed to fit observations. Instead the parameters have been set either to physically reasonable values based on observations or to values which, when used, have been found to give good fits to observations in previous works. As a result, the fits are remarkably good and, although far from conclusive, seem to validate both the chemical evolution model used (the CPM08 model) and the stellar population model adopted (GRASIL). Only two parameters were tuned in this work to match the observations. For the irregular galaxies in order to fit the MIR part of the SEDs of local dwarf irregulars the optical depth of the molecular clouds (MCs) in which the stars are born, needed to be reduced to a lower optical depth than the MCs in other starforming galaxies. It is not possible to say whether the improved fit requires a lower MC mass or a larger $\mathrm{MC}$ radius because the 
optical depth is dependent on both the mass and the radius. For the elliptical galaxies, to match the large amount of reprocessing observed in the SEDs of the SCUBA galaxies in the SHADES dataset, the time spent initially by the young stars in the dense MCs was increased to a larger value than that found for local normal starforming galaxies.

Also investigated are the potential errors you could expect if instead of following the evolution of the dust component of the ISM, two common assumption are adopted. These errors are given as the difference between our fiducial models with the detailed dust treatment and the models using the two simplifications. They will only be correct for the models presented here and not for any real galaxy in particular. However the chemical evolution models have been thoroughly tested against observations in previous works and, in this work the SEDs have been shown to be broadly consistent with the SEDs of the morphological type modeled. In addition, in all cases, the models generated using the dust evolution of CPM08 fit the observations better than the models generated when the two assumptions are adopted (figures $1 \mathrm{~b}, \mathrm{c}$ and $\mathrm{d}$ ). Therefore the errors presented in this work can be viewed as an example of the magnitude of errors that could be expected if the two assumptions are adopted in spiral, irregular and elliptical galaxies. In particular it has been shown that, for spiral galaxies, the errors that could be expected are small and decrease with the age of the galaxy (see figure 2a). This is unsurprising, since these assumptions have been developed based on observations of the Milky Way and normal local starforming galaxies. For irregular and elliptical galaxies, however, it has been shown that, potentially, the errors introduced by adopting the assumptions could be much larger (figures $2 \mathrm{~b}$ and $2 \mathrm{c}$ ). Of the assumptions it has been shown that, in all galactic types, the largest errors will be introduced if a relationship for the dust-togas ratio with the metallicity is adopted, instead of using a full dust evolution model to calculate this quantity. These errors would be largest in the IR part of the spectrum.

\section{Acknowledgments}

This work was supported through a Marie Curie studentship for the 6th Framework Research and Training network MAGPOP, contract number MRTN-CT-2004-503929.

\section{References}

Bauer, A. E., Drory, N., Hill, G. J., \& Feulner, G. 2005, ApJ, 621, L89

Calura, F., Pipino, A., \& Matteucci, F. 2008, A\&A, 479, 669

Clements et al., 2008, MNRAS, 387, 247

Dale, D. A. \& Helou, G. 2002, ApJ, 576, 159

Dale D. A., Gil de Paz A., Gordon K. D., et al. 2007 ApJ, 655, 863

Eales S., Lilly S., Webb T., Dunne L., Gear W., Clements D., \& Yun M., 2000, AJ, 120, 2244

Granato G. L. et al., 2000, ApJ, 542, 710

Hammer, F., Flores, H., Elbaz, D., Zheng, X. Z., Liang, Y. C., \& Cesarsky, C. 2005, A\&A, 430, 115

Hauser, M. G. \& Dwek, E. 2001, ARA\&A, 39, 249

Iglesias-Pramo, J. et al. 2007 ApJ, 670, 279

Panuzzo, P., Granato, G. L., Buat, V., Inoue, A. K., Silva, L., Iglesias-Pramo, J., \& Bressan, A 2007 MNRAS, 375, 640

Lilly, S. J., Eales, S. A., Gear, W. K. P., et al. 1999, ApJ, 518, 641

Popescu, C. C. \& Tuffs, R. J., 2005 AIPC, 761, 155P

Silva, L., Granato, G. L., Bressan, A., \& Danese, L., 1998, ApJ, 509, 103

Vega, O., Clemens, M. S., Bressan, A., Granato, G. L., Silva, L., \& Panuzzo, P. 2008, AछA, 484,631 